\title{
KGPMH: A hybrid approach for solving a novel facility location problem
}

This paper was downloaded from TechRxiv (https://www.techrxiv.org).

\section{LICENSE}

CC BY 4.0

SUBMISSION DATE / POSTED DATE

$25-10-2021 / 28-10-2021$

\section{CITATION}

DehghanChenary, Maryam; Ferdowsi, Arman; Jolai, Fariborz; Tavakkoli-Moghaddam, Reza (2021): KGPMH: A hybrid approach for solving a novel facility location problem. TechRxiv. Preprint. https://doi.org/10.36227/techrxiv.16866895.v1

DOI

10.36227/techrxiv.16866895.v1 


\title{
KGPMH: A hybrid approach for solving a novel facility location problem
}

\author{
Maryam Dehghan Chenary*, Arman Ferdowsi ${ }^{\dagger}$, Fariborz Jolai*, Reza Tavakkoli-Moghaddam* \\ ${ }^{*}$ School of Industrial Engineering, University of Tehran \\ maryamdehghan1374@gmail.com, \{fjolai, tavakoli\}@ut.ac.ir \\ †ECS Group, TU Wien \\ aferdowsi@ecs.tuwien.ac.at
}

\begin{abstract}
The focus of this paper is to propose a bi-objective mathematical model for a new extension of a multi-period $p$ mobile hub location problem and then to devise an algorithm for solving it. The developed model considers the impact of the time spent traveling at the hubs' network, the time spent at hubs for processing the flows, and the delay caused by congestion at hubs with specific capacities. Following the unveiled model, a hybrid meta-heuristic algorithm will be devised that simultaneously takes advantage of a novel evaluation function, a clustering technique, and a genetic approach for solving the proposed model. The experiments validate that the proposed algorithm outperforms better than several state-of-the-art algorithms. They also show the decisive effect of two considerable factors: congestion and service time.
\end{abstract}

Index Terms-Combinatorics optimization, Facility location problem, Heuristic approach

\section{INTRODUCTION}

In network and graph theory, designing a network $G$ might be referred to as the problem of selecting several nomination edges (i.e., connections) between the vertices of $G$ to curtail the sum of constructions and travel time. This paper is going to tackle one of the fundamental categories of network designing problems called Facility Location Problem $(F L P)$. This subject plays a decisive role in many research areas, such as structuring postal services [1], controlling air traffic [2], [3], scheduling manufacturing factories [4], supply chain warehouses [5], and even analyzing computer networks [6] [7], [8]. Roughly speaking, an FLP seeks to locate the number of facilities (i.e., hub vertices) that aim to satisfy the demands of clients (the remaining nodes) provided that several constraints hold [9], [10]. Consequently, it is about designing a transportation network that can optimize energy efficiency and commodity shipping costs as accurately as possible.

Therefore, the goal of an FLP is to plan a distribution flow network. The problem's input is the set of facilities and customers (i.e., set of vertices), and the output is the exact defined location of facilities and the way each customer is allocated to these facilities so that the facilities' services can be used optimally. In this fashion, the network scheme of an FLP needs to be designed in a way that a specific and limited number of nodes are selected as hubs. These hubs form a complete graph and have the duty to merge flows from origin nodes and deliver them to destination nodes to satisfy the desired demand of each vertex (i.e., customer) as well as to minimize the total cost and service time. Hence, the primary argument is selecting suitable locations from the available places to establish facilities there. These hub vertices will then characterize the flow paths and organize the transit among sources and destination spots.

There are two basic hypotheses in the study of this problem that can be summarized as follows: $(i)$ there is no direct route between two non-hub nodes, and ( $i i$ ) each non-hub node has to be allocated to precisely one hub vertex. The latter is referred to as the single-allocation condition [11]. We suppose that the total number of nodes and the number of hubs are fixed and denoted by $n$ and $p$. Since each node has a demand that has to be satisfied, the purpose is to locate the hubs responsible for providing services and fast transfer of demands in the best way so that all demands are satisfied, and also the transportation cost achieves its lowest possible value.

One crucial aspect of FLPs is that hubs can have capacities. In this case, the problem's model needs to have capacity constraints that guarantee that the total demands of clients allocated to a hub does not exceed that hub's capacity.

Another critical factor of the $p$-hub location problems is $d y$ namism. Clearly, in the real-world, the decision is not limited to just one period of time (e.g., yearly), meanig that many structural operations, decisions variable, problem parameters, budgets, and many other influencing variables may change over time. To be more precise, early decisions may change under various factors. For instance, demands (i.e., flows) can differ in different periods. In dynamic FLPs, current facilities are used in each period, or new facilities are made. Dynamic location problems consist of two categories: One is to change the location decisions in each period, and one is to make the strategic location decisions initially. In the second case, the location decisions are not allowed to change during periods [12].

An extra property of a hub location problem, which can be investigated, is the mobility of hubs. Hubs can be gathered into two groups of fix and mobile hubs. Mobile hubs are capable of movement. That is, they can move to other places if needed. One can trivially think of several applications and examples of mobile hub problems, including emergency services, fire stations, and blood collection centers. These queries can be created during a natural incident and be relocated in the case of necessity to offer facilities to the applicants with 
fewer costs and less time spent [13]. One important argument is that mobile hubs should be able to move among other vertices. Accordingly, before these movements can take place, infrastructures between nodes have to be provided. Therefore, these movement infrastructures are provided in the first period and can be used in other periods.

On the other hand, most studies about facility location problems focus mainly on reducing costs and ignore possible other goals like delivery time. Nevertheless, because clients and customers prefer to receive facilities from a center that offers facilities with fewer costs and also less service time, in recent years, increasing competition between service centers (e.g., factories) has been accrued in terms of paying attention to the service and waiting time for providing services.

Service time is a vital and inseparable component of FLPs, and in its absence, the designed network may not be feasible in terms of the desirability of the service level. The service time can be divided into two components: $(i)$ the time it takes to reach streams from non-hub nodes to hubs and (ii) time elapsed at hubs for proceeding the flows. We call the latter the handling time. Handling time at a hub depends on the capacity and congestion level and the capacity usage percentage of that hub. Delay may occur during the flow process at hubs. This delay is because the hub is working with almost its total capacity. In this case, the hub is congested. Consequently, having a limited capacity at hubs in transportation networks causes congestion that leads to increasing the waiting time for receiving the services and delays the service time as well. Therefore, creating a transportation network based on decreasing congestion is a fundamental issue. Generally speaking, the congestion level is calculated by the two following factors: (i) the amount of flow that hubs need to process and (ii) maximum hub capacity [14].

Main contribution: First of all, this work generalizes one of the basic classes of a $p$-mobile hub location problem by adding (considering) hubs' congestion and the service time factor. To the best of our knowledge, this is the first time these two crucial factors are being considered for such a problem. To be more specific, this paper considers a problem consisting of the dynamism factor, the mobility of hubs, service time limit, capacity, and congestion. In this regard, the aim is to design a transportation network and (near) optimally locate the mobile facilities such that the transportation cost, cost of creating and transporting hubs, cost of establishing mobile infrastructure, the ready time, and congestion level at hubs in three different periods all be minimized. These considerations faced us with devising a (complex) new biobjective Mixed-Integer optimization problem. Unfortunately, it is worth mentioning that reducing costs and reducing the delivery time at the same time usually contrast with each other. Therefore, it is very challenging to create a proper equivalence between these two issues.

Such an FLP model falls in NP-hard problems. Accordingly, no exact algorithm likely exists that returns the optimal answer, especially in large-scale models, at a reasonable time. As a result, one can argue that (meta) heuristic algorithms can play a fundamental role in solving this problem. We, therefore, propose a novel hybrid meta-heuristic method for solving the recommended problem. Our hybrid approach consists of i) creating a set of initial feasible solutions, ii) devising a method for evaluating the solutions based on a novel evaluation function, converting the solutions into a matrix representation, and employing a clustering algorithm, and iii) creating new sets of solutions based on a genetic approach.

We managed to evaluate our algorithm under variant conditions comprehensively. We tested our algorithm based on the well-known Australia Post dataset under considering different conditions, including different numbers of nodes, different numbers of algorithm's iterations, different numbers of initial solutions, different numbers of hubs, etc. We compared our results with those obtained by several other state-of-the-art algorithms, which are all among the well-known and commonlyused facility locations algorithms. We also compared the outcomes with the ones obtained by the very powerful solver CPLEX. As the results show, our method outperforms all the comparable algorithms in all experimental cases in terms of the running time as well as the solutions' quality. Furthermore, we also investigated the role of considering the mobility of hubs, congestion, and service time.

Paper organization: Section II gives a brief history of FLPs and some related state-of-the-art research work. Section III models and formulates the proposed $p$-mobile hub location problem considering congestion and service time. Section IV is dedicated to presenting our hybrid meta-heuristic algorithm. Section V, finally, brings forward the experimental results.

\section{LITERATURE REVIEW}

In 1987, O'kelly introduced the single-allocation $p$-median location problem [15], which attracted many other researchers to study, extend and develop this subject. After that, Campbell [16] introduced a mathematical model for the single-allocation $p$-hub median problem in which the limited capacity of incoming flow to hubs was considered. Capacity is an essential property in hub location problems (HLP) that can be assumed for both hubs and flow transfer edges. One can think of considering some different capacity levels for each hub so that each of them has to pick one capacity level. In this fashion, Correia et al. [17] have developed a mathematical model for the single-allocation HLP with capacity constraints in terms of several capacity levels.

On the other hand, another argument might be that one can face a congestion situation in transportation systems since high or increased demand for services may occur. This case happens when an intended hub works near its full capacity, and in this situation, we say that the hub is congested. The congestion issue was introduced by Grove and O'Kelly [18]. It is known that the queue theory has been used for considering congestion in most papers. Karimi et al. [19] addressed the single allocation hub-and-spoke network design under hub congestion. They modeled the network through a bi-objective non-linear mixed-integer programming. The model was developed based on a general GI/G/G queuing system. They managed to solve 
their proposed model using a learning-based meta-heuristic based on NSGA-II, k-means clustering method, and an Iterated Local Search. Ghodratnama et al. [20], on the other hand, developed a new hub location with considering congestion and production schedule. They developed a bi-objective hub location model to deal with the problem. In their consideration, the first objective is to minimize the total cost, and the second objective is to minimize the waiting time for processing goods by modeling the system as a queuing network. In order to solve the bi-objective problem, they devised a novel hybrid Goal Attainment and LP Metric approach.

On the other hand, however, Alumur et al. [14] modeled the service time and congestion constraints in single and multiallocation HLP by avoiding the queue theory. Rodriguez et al. [21] stated that service time consists of traveling time through network and time spent in hubs. In most cases, time spent in hubs is considered by the waiting time in a queue. However, like what Alumur et al. [14] explained, we calculate the time spent at hubs by considering the capacity and congestion levels of hubs and also the hub's capacity percentage of use.

Another important argument is that in real-world cases, decisions are not made for only one specific period. During the implementation of extensive structural operations, sometimes planning horizons needs to be considered and predicted by particular periods. For instance, demand (i.e., flow) may differ in the transportation problem in various periods. The subject of considering different periods in this kind of problem was first introduced by Melo et al. [22]. Alumur et al. [23] presented a mathematical model for a multi-period (i.e., dynamic) HLP with single and multiple allocations and considering modular capacities. Correia et al. [24] brought forward a mathematical model for a multi-period HLP considering stochastic flows and limited capacity.

One can also investigate this problem from another critical viewpoint: mobility of hubs. The intuition is that mobile facilities can move to other locations without any establishment costs to satisfy the client's demands. Halper and Raghavan [25] first introduced the mobile hub problem. Lei et al. [26] presented a two-stage mixed-integer stochastic approach for routing mobile facilities with stochastic demands in a discrete number of scenarios. In the first stage, a decision about whether the mobile facilities are used or not and where and when they are used is made. In the second stage, a decision about how clients allocate to these facilities is made. Güden and Süral [27] studied a mobile FLP in the field of railway construction so that mobile facilities can move through railways. Furthermore, Bashiri et al. [28] presented and studied the single-allocation $p$-mobile hub location problem in the dynamic environment. This was a tremendous breakthrough because of combining two significant factors: dynamism and mobility of hubs. In their model, hubs can move instead of opening and closing. They compared their proposed model with the classical dynamic hub location problem. They were succeeded in showing that the model is much more efficient, and the total cost significantly decreases whenever hubs can move easily.It is worth mentioning that they used the genetic algorithm and the local search approach to solve their model. Qi et al. [29] introduced a mobile hub routing problem that considers service time related to the demand rate and fairness constraints. They developed a mixed-integer programming model for their problem and used a meta-heuristic algorithm for solving it. Beside, Mokhtarzadeh et al. [30] investigated a multi-objective p-mobile hub location problem with the depreciation cost of hub facilities. The authors then developed a hybrid of k-medoids, a clustering algorithm, with two metaheuristic algorithms to solve the presented problem.

We refer the reader to [31], [32], [33], and [34] to see comprehensive surveys on this problem and its different aspects.

Let us point out that one can also investigate the context problem from the perspective of the solution approach. For instance, there is a small number of approaches that are based on optimally solving the mathematical programming model; See [35], [36], [37], and [38]). Nevertheless, as mentioned, since the under-study problem is an NP-hard one, most of the solution approaches that have been proposed so far follow (meta) heuristic manners; See, for example, [39], [40], and [41].

On the other hand, it turned out to be unlikely to find a straightforward optimization since the problem lies in multiobjective optimization problems. To be more precise, in this kind of problem, different objective functions usually conflict with each other, meaning that minimizing one of them can lead to maximizing the other one. This obstacle led to the emerging a new concept of the optimal solution, the Pareto solutions. As we will figure out later, this concept will help us to deal with multi-objective optimization. In consequence, although all the heuristic approaches, which have been developed so far, work based on different methodologies, they almost aim at finding the Pareto optimal solutions. For instance, [42] provides a genetic-based for obtaining Pareto solutions, while [43] proposes a dynamic aggregation technique for doing this. However, one of the recent approaches that some researchers have recently noticed and used is to apply a clustering method to somehow evaluate solutions with simultaneous respect to each objective function: See [44] and [45].

We end this section by referring the interested reader to [46] for attaining a comprehensive vision of this problem.

\section{PROBLEM DEFINITION AND THE PROPOSED MODEL}

In this section, we aim to introduce the basic assumptions of our under-study FLP. Afterward, we bring forward the mathematical formulation of the devised model based on the explained assumptions.

\section{A. Problem assumptions}

The classic form of a hub location problem consists of three following basic assumptions:

- No direct route between a origin and destination points is allowed to exist.

- Connection between hubs are complete. 
- Flow transfer between each pair of hubs includes a discount factor $\alpha$ which causes to save the cost of mass transit of goods.

This paper not only uses the assumptions related to the classic form but also considers several other assumptions as follows: [14] and [28]

- single-allocation strategy is considered.

- The number of hubs in each period is fixed and equal to $p$.

- Each node has the potential of being a hub.

- Hubs mobility infrastructures are created in the first period and can be used in the next periods

- If there is any mobile infrastructure, hubs can move to other vertices in the next periods otherwise, a new hub node should be set up.

- Each hub can tolerate a certain amount of incoming flow, and this specifies the limited capacity topic. Each hub has a specific capacity level.

- A service time limit exists for sending flow among origin and destination nodes. That is to say, each pair of nodes has to gain services within this given service time limit.

- A general service time limit exists for the entire network.

- Service time between two nodes is obtained by the travel time on the network plus the handling times at hubs.

- The total cost is regarded and computed for locating hubs having specific capacities, flow transfer cost, creating mobile infrastructures and hubs' transfer costs.

- If transportation costs satisfy the triangle inequality, then all the traffic is directed by at most two hubs, since the hub network is a complete graph.

- Traveling time is symmetric.

\section{B. Mathematical formulation}

This paper presents a bi-objective model for a singleallocation multi-period problem. The proposed model is based on the model which Bashiri et al. [28] and Alumur et al. [14] have introduced. Before depicting the mathematical model, we first draw your attention to introducing the parameters and decision variables of the model.

1) Sets, parameters and decision variables: Sets:

$N \quad$ Set of vertices

$C \quad$ Set of capacity levels of hubs

$G \quad$ Set of congestion levels of hubs

$T \quad$ Set of periods

\section{Parameters:}

$\alpha \quad$ Discount rate

$p \quad$ Number of hubs

$W_{i j} \quad$ Flow sending from node $i$ to node $j$

$O_{i}=\sum_{j \in N} W_{i j} \quad$ Total flow emanate from $i$

$D_{j}=\sum_{i \in N} W_{i j} \quad$ Total flow entering $j$

$F 1_{k, c}^{t} \quad$ Fixed cost for constructing hub $k$ with capacity $c$ in period $t$

$F 2_{i, k}^{t} \quad$ Cost of transferring a mobile hub $k$ from node $i$ to node $k$ in period $t$

$C 1_{i, k}^{t} \quad$ Cost of flow transfer between node $i$ and hub $k$ in period $t$
$C 2_{k, l}^{t} \quad$ Cost of constructing a route between hubs $k$ and $l$

$t_{i j}^{t} \quad$ Traveling time between nodes $i$ and $j$

$\Delta^{c} \quad$ Handling time of a hub with a capacity level $c \in C$

$\sigma^{t} \quad$ Service time limit in period $t$

$\Gamma^{c} \quad$ Capacity of a hub with a capacity level $c \in C$

$\gamma^{g} \quad$ The upper limit of the used capacity (in terms of percentage) for a hub congested at level $g \in G$

$\tau_{g} \quad$ Congestion factor (delay in time, associated with the congestion level $g \in G$ )

\section{Decision variables:}

$X_{i, k}^{t} \quad 1$ if in period $t$ vertex $i$ is allocated in hub $k ; 0$, otherwise

$Z 1_{k}^{t} \quad 1$ if in period $t$ vertex $k$ is a non-mobile vertex; 0 , otherwise

$Z 2_{i, k}^{t} \quad 1$ if in period $t$ hub $i$ transfers to hub $k$; 0 , otherwise

$Y e_{k, c, g}^{t} \quad 1$ if in period $t$ hub $k$ has capacity $c$ and congestion level $g$; 0 , otherwise

$Y 1_{i, k, l, j}^{t} \quad 1$ if in period $t$ the flow from node $i$ and node $j$ is established from hubs $k$ and $l$; 0 , otherwise

$R_{k, l} \quad 1$ if mobile infrastructure between nodes $k$ and $l$ is established; 0 , otherwise

$Y 2_{k}^{t, t-1} \quad 1$ if node $k$ is hub in periods $t-1$ and $t ; 0$, otherwise

$r_{k}^{t} \quad$ ready time in hub $k$ in period $t$

Be advised that the service time between two nodes depends on the traveling time through network plus the handling time at hubs, where the handling time at hub $k$ depends on the capacity and congestion level of $k$, itself. On the other hand, congestion level at $k$ depends on the percentage of $k$ 's capacity utilization. Let $C=\left\{c_{1}, c_{2}, \ldots, c_{m}\right\}$ be the set of capacity levels of hubs where, capacity level $c_{i}$ is higher than the capacity level $c_{i-1}$ for every $i \in\{1,2, \ldots, m\}$. Then $\Gamma=\left[\Gamma^{c_{1}}, \Gamma^{c_{2}}, \ldots, \Gamma^{c_{m}}\right]$ and $\Delta=\left[\Delta^{c_{1}}, \Delta^{c_{2}}, \ldots, \Delta^{c_{m}}\right]$ are two vectors such that $\Gamma^{c_{i}}>\Gamma^{c_{i-1}}$ and $\Delta^{c_{i}}>\Delta^{c_{i-1}}$ $(1 \leq i \leq m)$. By this notation, we mean that a hub with a higher capacity level has to contain a higher capacity and handling time. Let $G=\{1,2, \ldots, l\}$ be the set of congestion levels. $\gamma=\left[\gamma^{1}, \gamma^{2}, \ldots, \gamma^{l}\right]$ is a vector, where $\gamma^{i}>\gamma^{i-1}$ $(1 \leq i \leq l)$. That is, a hub with a higher congestion level can use the higher amount of its capacity rather than a hub with a lower congestion level. On the other hand, $\tau_{i}>\tau_{i-1}$ $(1 \leq i \leq l)$ and it means, the higher the congestion level of a hub is, the more delay in the hub for processing flow occurs. $r_{k}$ represents the ready time for the hub $k$ and it is equal to the latest arrival or departing time of all flow arriving at or exiting from $k$.

2) Mathematical problem modelling: By inspiring from [14], [28], in what follows, we present the mathematical formulation of our proposed model which explains a $p$ mobile hub location problem with considering service time and congestion at hubs. We name the model PMHM that stands for $p$-mobile hub model. Let $i, l, k \in N, c \in C, g \in G$ and $t \in T$. PMHM consists of two objective functions along with 
20 constraints as follows:

$$
\begin{aligned}
& \operatorname{Min} \sum_{t, i, j, k} C 1_{i, k}^{t} W_{i, j}^{t} X_{i, k}^{t}+\sum_{t, i, k, l, j} \alpha C 1_{i, k}^{t} W_{i, j}^{t} Y 1_{i, k, l, j}^{t} \\
& +\sum_{k, l} C 2_{k, l} R_{k, l}+\sum_{t, i, k} F 2_{i, k}^{t} Z 2_{i, k}^{t}+\sum_{t, k, c, g} F 1_{k, c}^{t} Y e_{k, c, g}^{t}
\end{aligned}
$$

$\operatorname{Min} \sum_{t, k, c, g} \gamma^{g} Y e_{k, c, g}^{t}+\sum_{t, k} r_{k}^{t}$

subject to:

$$
\begin{aligned}
& Z 2_{i, k}^{1}=0, \forall i, k \\
& X_{k, k}^{t}=Z 1_{k}^{t}+\sum_{i, i \neq k} Z 2_{i, k}^{t}, \forall t, k \\
& \sum_{k} X_{k, k}^{t}=p, \forall t \\
& X_{i, k}^{t} \leq X_{k, k}^{t}, \forall t, i, k \\
& \sum_{k} X_{i, k}^{t}=1, \forall t, i \\
& Z 2_{k, i}^{t} \leq X_{k, k}^{t-1}, \forall t, k, i, k \neq i \\
& Z 2_{k, l}^{t} \leq R_{k, l}, \forall t, k, l, k \neq l \\
& X_{i, k}^{t}+X_{j, l}^{t}-1 \leq Y 1_{i, k, l, j}^{t}, \forall t, i, k, l, j \\
& Y 1_{i, k, l, j}^{t} \leq X_{i, k}^{t}, \forall t, i, k, l, j \\
& Y 1_{i, k, l, j}^{t} \leq X_{j, l}^{t}, \forall t, i, k, l, j \\
& Y e_{k, c, g}^{t}=Z 1_{k}^{t}-Y 2_{k}^{t, t-1}, \forall t, k \\
& Z 1_{k}^{t}+Z 1_{k}^{t-1}-1 \leq Y 2_{k}^{t, t-1}, \forall t, k \\
& Y 2_{k}^{t, t-1} \leq Z 1_{k}^{t}, \forall t, k \\
& Y 2_{k}^{t, t-1} \leq Z 1_{k}^{t-1}, \forall t, k \\
& \sum_{c, g} Y e_{k, c, g}^{t}=X_{k, k}^{t}, \forall t, k \\
& Z 1_{k}^{t}+\sum_{i, i \neq k} Z 2_{k, i}^{t} \leq 1, \forall t, k \\
& \sum_{i} o_{i}^{t} X_{i, k}^{t} \leq \sum_{c, g} \Gamma^{c} \gamma^{g} Y e_{k, c, g}^{t}, \forall t, k \\
& r_{k}^{t} \geq t_{i, k}^{t} X_{i, k}^{t}, \forall t, k, i \\
& r_{k}^{t}+\sum_{c, g} \Delta^{c} \tau_{g} Y e_{k, c, g}^{t}+t_{k, l}^{t}+\sum_{c, g} \Delta^{c} Y e_{l, c, g}^{t}+r_{l}^{t} \leq \sigma^{t} \\
& , \forall t, k, l \\
& X_{i, k}^{t}, Z 1_{k}^{t}, Z 2_{k, i}^{t}, Y 1_{i, k, l, j}^{t}, Y e_{k, c, g}^{t}, R_{k, l}, Y 2_{k}^{t, t-1} \in\{0,1\} \\
& , r_{k}^{t} \geq 0 ; \forall t, i, k, l, j
\end{aligned}
$$

Objective function (1) minimizes the total cost of transportation, constructing mobile infrastructures, establishing hubs and transportation of hubs. Objective function (2) minimizes the congestion level and ready time in hubs. Equations (3)-(22) are constraints of PMHM. Constraints (3) guarantee that no hubs are transported in the first period. Constraints (4) make sure that in each period and vertex, one hub can be constructed or transferred to another vertex. Constraints (5) show the number of hubs in each period. Constraints (6) say that neither of the non-hub nodes is adjacent. Constraints (7) emphasize that the allocation strategy is single. Constraints (8) show that if $k$ is a hub in period $t-1$, it can move to hub $i$ in period $t$. Constraints (9) imply that if a mobile infrastructure is available between two nodes, a hub can move between these nodes. Constraints (10)-(12) express the route between pair of nodes. Constraints (13)-(16) are used for hub infrastructure differentiation. Constraints (17) explain that each hub can only have one capacity level and one congestion level. Constraints (18) say that in each period, a hub can be established or moved to other vertices. Capacity constraints are inflicted by constraints (19). For each hub in each period, the congestion level is determined simultaneously by these constraints. The higher the percentage of use of a hub's capacity (i.e., the maximum inflow that this hub processes), the higher its congestion level is. Constraints (20) consider a bound for the ready time. The value of the ready time for a hub in each period has to be greater than or equal to the longest time for collecting flows from all the nodes allocated to that hub. Constraints (21) guarantee that the service time in each period does not exceed service time limit $\sigma$. These constraints include two following components of the total service time: $(i)$ transportation time, which is independent from congestion level in hubs, and (ii) the handling time at hubs, which depends on congestion. Traveling time between a pair of origin and destination nodes is obtained by the sum of the ready time at each hub, handling time at hubs, and traveling time among the hubs. It's worth mentioning that delay throughout congestion only sticks at the first hub on the route since capacity constraints are only inflicted on the inflow. Constraints (22) show the domain constraints (see [14], [28]).

\section{SOLUTION APPROACH}

In this section, we are going to propose our hybrid metaheuristic algorithm that aims at discovering a (near) optimal solution to the model presented in the previous section. In a nutshell, our algorithm first creates a set of random feasible solutions to the PMHM. It then accomplishes a clustering task and evaluates the solutions by creating a matrix representation and employing the famous K-means algorithm. This batching helps us cluster solutions so that the solutions with approximately similar ranks belong to the same classes. In this fashion, a large number of solutions will be pruned instantly, and many of them belong to low-quality clusters will be omitted. Afterward, a genetic approach will be employed to generate new solutions based on the previously obtained highquality remained solutions. It is natural to expect to obtain a (near) optimal solution by repeating this procedure. In the sequel, we dive into expressing the proposed method.

Nevertheless, before diving into proposing our approach, one crucial argument needs to be taken care of. As we will see, this issue plays a pivotal role in our further discussion, especially in evaluating the obtained solutions. 
Recall that we are facing a bi-objective optimization problem. The challenging issue while dealing with these problems, or in general multi-objective optimization problems, is that the objective functions might conflict, meaning that, the variant solutions to these problems weigh up different goals. To be more specific, a feasible solution that minimizes all objective functions simultaneously does not typically exist in our understudy bi-objective optimization problem. Therefore, by a solution optimal, one can refer to as Pareto optimal solutions: solutions that cannot be improved in any of the objectives without degrading at the other objectives. This fact (besides the NP-hardness of the problem) somehow means that we may induce failure to discover the optimal solution. Accordingly, in this kind of problem, one might think of looking for solutions that hold the respective balance between goals. To be more precise, solutions must be found that do not limit us to optimizing just one objective function. Consequently, in this fashion, most of the research work in this area uses the notion of domination [47]: Let $s_{1}$ and $s_{2}$ be two solutions to a biobjective problem. $s_{1}$ is said to dominate $s_{2}$ if two following conditions hold:

1) Neither of the objective functions' values for $s_{1}$ shouldn't have worse value of the objective functions for $s_{2}$.

2) For at least one objective function, $s_{1}$ has to have strictly better value than $s_{2}$.

The domination concept enables us to make a favorable balance among the obtained solutions. As will be discussed later, we take advantage of this notion for further evaluating the solutions to ultimately propose a method that aims to discover (near) Pareto optimal solutions. In this vein, we shall first find a way to represent and create (initial) solutions to the study model.

\section{A. Representing and creating a random feasible solutions to PMHM}

This work considers three levels for both capacity and congestion for each hub. These levels are represented by the integer values 1,2 , and 3 . It also considers three periods of time $(T=\{1,2,3\})$. In what follows, we present a way for expressing a solution as well as creating a random feasible solution of PMHM for each period $t \in T$.

For a specific time period $t \in T$, we represent a feasible solution $s^{(t)}$ to PMHM by an $5 \times(n+p)$ matrix where $n$ and $p$ are the number of nodes and the number of hubs, respectively. In this fashion $s_{i, j}^{(t)}$ is said to be the element placed in the row $i$ and column $j$ of $s^{(t)}$. The first row of this matrix helps us to create a solution and the second to the fifth rows helps us presenting it.

We first express the way we create this matrix. Afterward, we explain how this obtained matrix can perfectly represent a random feasible solution $s^{(t)}$.

- First row: the first $n$ entries are filled by $n$ random real numbers between 0 and 1 . Elements located at positions (columns) $n+1$ to $n+p-1$ are filled out with random integer numbers between 1 and $n-1$ and the last element $((n+p)$-th column) gets the value of $n$.
- Second row: suppose that, $j_{1}$ is the index of a column that contains the maximum element among the first $n$ columns of $s^{(t)}$ 's first row and let $s_{2, j_{1}}^{(t)}=1$. Now assume that $j_{2}$ is the index of a column that contains the next maximum element among the first $n$ columns of $s^{(t)}$ 's first row and let $s_{2, j_{2}}^{(t)}=2$. We continue this procedure until all the first $n$ columns of the second row are set by the integer numbers 1 to $n$. The $(n+1)$-th to $(n+p)$-th columns of this row get exactly the values of the $(n+1)$-th to $(n+p)$-th columns of the first row.

- Third and Forth row: assume that $A=\bigcup_{j=n+1}^{n+p} s_{1, j}^{(t)}$. $s_{3, j}^{(t)}$ and $s_{4, j}^{(t)}$ for all $j \in\{1,2, \ldots, n+p\}$ are defined as follows:

$$
\begin{aligned}
& s_{3, j}^{(t)}= \begin{cases}\text { random integer number between } 1 \text { and } 3, & \text { if } j \in A \\
0 \quad, & \text { otherwise }\end{cases} \\
& s_{4, j}^{(t)}= \begin{cases}\text { random integer number between } 1 \text { and } 3, & \text { if } j \in A \\
0 \quad, & \text { otherwise }\end{cases}
\end{aligned}
$$

- Fifth row: we define entries of this row as follows:

$$
s_{5, j}^{(t)}=\left\{\begin{array}{cc}
r^{(t)}, & \text { if } j \in A \\
s_{2, j}^{(t)}, & \text { otherwise }
\end{array},^{(},\right.
$$

where $A$ is the set defined in the previous item and $j \in$ $\{1,2, \ldots, n+p\}$.

We claim that the introduced matrix can perfectly represent a feasible solution: The second row of $s^{(t)}$ specifies hub nodes and the allocation of non-hub nodes to hubs. $s_{2, n+1}^{(t)}, \ldots, s_{2, n+p}^{(t)}$ represent the indices of hub node. To be more specific, $s_{2, s_{2, j}^{(t)}}^{(t)}$ is a hub vertex for every $j \in\{n+$ $1, \ldots, n+p\}$. On the other hand, each of the first $n$ elements of this row, which is not a hub, is allocated to the first hub placed at the right-side of that element. The third and fourth rows respectively express the capacity and congestion levels of hubs. Furthermore, the fifth row represents the ready time value for each hub. With possession of all solution matrices $s^{(t)}(t \in T)$, all the decision variables introduced in the previous section can be derived.

Fig. 1 shows a sample of a solution matrix of a specific time period that contains 7 nodes and 3 hubs. As we can figure out, nodes 5,2 , and 3 are hubs. Node 6 is allocated to hub 5 . Node 7 is allocated to hub 2, and nodes 1 and 4 are allocated to hub 3 . In addition, for example, hub 5 has the capacity and congestion level 1. Fig. 2 represents the network scheme corresponding to the solution shown in Fig. 1. Suppose that the weights over the edges in Fig. 2 show the traveling time between each pair of vertices, and also, weights over the hub vertices show the handling time. Ready time, for example, for hub 3 (i.e., $r_{3}$ ) equals 5 . The longest service time in this network is $14.5 \mathrm{~h}$.

One important issue to mention is that since our model is supposed to consider three periods for PMHM $(T=\{1,2,3\})$, each solution has to contain all the three periods' information. Accordingly, we consider each solution to PMHM as a set $s=\left\{s^{(1)}, s^{(2)}, s^{(3)}\right\}$ where, each $s^{(t)}(t \in\{1,2,3\})$ is a solution for the period $t$. Furthermore, recall that the first row 


\begin{tabular}{|c|c|c|c|c|c|c|c|c|c|}
\hline 0.2852 & 0.3287 & 0.1622 & 0.6813 & 0.9193 & 0.4615 & 0.6030 & 2.0000 & 4.0000 & 7.0000 \\
\hline 6.0000 & 5.0000 & 7.0000 & 2.0000 & 1.0000 & 4.0000 & 3.0000 & 2.0000 & 4.0000 & 7.0000 \\
\hline 0 & 1.0000 & 0 & 3.0000 & 0 & 0 & 2.0000 & 0 & 0 & 0 \\
\hline 0 & 1.0000 & 0 & 3.0000 & 0 & 0 & 2.0000 & 0 & 0 & 0 \\
\hline 0 & 3.0000 & 0 & 4.0000 & 0 & 0 & 5.0000 & 0 & 0 & 0 \\
\hline
\end{tabular}

Fig. 1: Representation of a random solution $s^{(t)}$

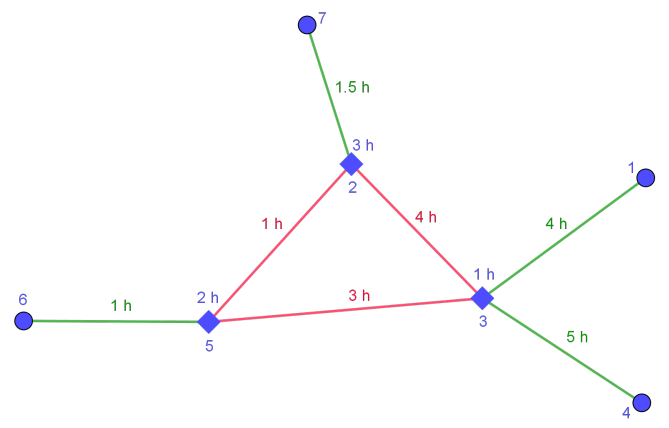

Fig. 2: Network scheme of the solution $s^{(t)}$ shown in Fig. 1 . Nodes 2, 3 and 5 are hubs and are fully connected.

is responsible for creating a solution by which the other rows will be defined. Accordingly, for each solution $s$, we denote the fist row of the matrix representation of $s$ as the leader row and denote it by $l(1,:)$.

\section{B. Evaluating the solutions}

This section plays a critical role in our research. In what follows, we first introduce a new function that enables us to evaluate each individual solution to another based on the well-known Chi-square distribution. Utilizing the proposed function and the domination notion, we then devise vector representation for each solution, which further helps us evaluate solutions more accurately using a clustering technique We coined the term VRCE for our vector representation clusteringbased evaluation method.

Regarding the evaluation matrix, we first let $f: S \rightarrow \mathbb{R}$ to be a monotonically non-decreasing function, such that $f(s(i))$ considers the number of solutions that $s(i)$ dominates and corresponds a real value number between 0 and 1 to that number. A very naive function $f$ can be defined in a way that for each solution $s(i), f(s(i))$ is the normalized value of the number of solutions that $s(i)$ dominates. Nevertheless, we introduce a novel function, which further leads to much more accurate outcomes. We employ the Chi-squared distribution, the cumulative distribution function for defining the function $f$. Mathematically speaking, given a solution $s \in S$, assume that $i$ is the number of solutions that $s$ dominates. We consider:

$$
f(s)=\frac{\gamma\left(\frac{1}{2}, \frac{i}{2}\right)}{\Gamma\left(\frac{1}{2}\right)},
$$

where $\Gamma(x)$ and $\gamma(y, z)$ are respectively the gamma and the lower incomplete gamma function [48]. It is easy to check that $f$ is a monotonically non-decreasing function with the range $[0,1]$.
We now introduce a way to generate a vector representation $v_{i}$ for each solution $s(i) \in S$. Let $v_{i}(1)=f(s(i))$. $v_{i}(2)$ and $v_{i}(3)$ will be created as follows. Intuitively, $v_{i}(2)$ and $v_{i}(3)$ show how much the first resp. second objective function's value can be improved by considering $s(i)$ over other solutions. Mathematically speaking, we have:

$$
\begin{aligned}
& v_{i}(2)=\frac{\sum_{s(j) \in \mathcal{D}} o b j^{(1)}(s(j))-|\mathcal{D}| \cdot o b j^{(1)}(s(i))}{|\mathcal{D}|}, \\
& v_{i}(3)=\frac{\sum_{s(j) \in \mathcal{D}} o b j^{(2)}(s(j))-|\mathcal{D}| \cdot o b j^{(2)}(s(i))}{|\mathcal{D}|},
\end{aligned}
$$

where $\mathcal{D} \subseteq S$ is the set of solutions that are dominated by $s(i)$, and furthermore, $o b j^{(1)}(s(i))$, for example, means the first objective value in terms of the solution $s(i)$.

Now, let $M=\left(v_{1}, \ldots v_{n}\right)^{T}$. Instinctively, matrix $M$ can be thought of as a dataset matrix, equipped with three features, whose each row is a vector representation of a solution that can perfectly reflect the goodness of that solution.

Right after achieving this matrix representation, the $K$ means algorithm will be applied so that to cluster the obtained solutions in terms of their similarities. To be more specific, by noticing the matrix $M$, the similarity between two solutions can be expressed based on:

- Similarity between the number of solutions they can dominate.

- Similarity between the amount of improvement they can establish on the first and second objective function.

In this vain, solutions with the same similarities are likely to belong to the same colonies. Considerably promising is that one can immediately conclude that two solutions in a same cluster are likely to dominate almost the same number of solutions, and moreover, they can decrease the objective functions by almost the same amount. To be more precise, this clustering can be thought of as an evaluation procedure. We end up this section with providing three following notations. Let $\mathcal{C}=\left\{C_{1}, \ldots C_{k}\right\}$ be $k$ clusters of solutions achieved by the $K$-means algorithm.

- We define the rank of a solution $s_{i} \in S$ as $r(i)=$ $\sum_{j=1}^{3} v_{i}(j)$.

- We refer to $w(j)=\sum_{i \in C_{j}} \frac{r(i)}{\left|C_{j}\right|}$ as the rank of the cluster $C_{j} \in \mathcal{C}$.

- $w^{i}$ denotes the rank of a cluster that contains the solution $s_{i}$. 


\section{Creating new generations of solutions}

To generate a new set of feasible solutions, which inherit the superior genomes of the parents (already obtained feasible solutions), a genetic approach is employed. However, considerably promising is that unlike the many naive genetic method used in almost many previous research, we wisely use the clusters obtained in Section IV-B for generating more accurate solutions. In a nutshell, the idea is that the probability of generating a new solution based on two parents (two solutions) that belong to a high-quality cluster shall be more than when they belong to lower quality cluster. Consequently, since the parents are often selected from high-quality clusters, it is very likely to expect the new solutions to get even better during the procedure.

The following repeatable procedure present the employed genetic algorithm. Be noted that two solutions will be picked as parents in each iteration, and two new solutions will be generated afterward.

- Repeat the following steps for a desired number of iterations.

- Pick two solutions $s, s^{\prime} \in S$ in such a way that the probability of choosing a solution $s_{i} \in S$ is equal to

$$
\frac{w^{i}}{\sum_{j=1}^{\left|w^{\mid}\right|} w^{j}}{ }^{1} \text {. }
$$

- Consider the leader rows of these solutions (i.e., $l_{s}(1$, : ),$\left.l_{s^{\prime}}(1,:)\right)$.

- Select one of the three following procedures with equal probability:

* Single-point crossover:

- A point on both $l_{s}(1,:)$ and $l_{s^{\prime}}(1,:)$ is picked randomly. We coin the term a crossover point for it. Genes to the right of that point are swapped between the two parent solutions.

- In this fashion we will have two new leader rows.

- Based on them and also the procedure explained in Section IV-A, we create two new solutions and add them to $S$.

* Two-point crossover:

- two crossover points are picked randomly on both $l_{s}(1,:)$ and $l_{s^{\prime}}(1,:)$. The bits in between the two points are swapped between the parent organisms.

- Same as what we described for the case of single-point crossover, we create two new solutions and add them to $S$.

\section{* Mutation:}

- We randomly choose two integer numbers $r_{1}$ and $r_{2}$ between 0 and $n$ so that to change $r_{1}$ genomes of the $l_{s}(1,:)$ and $r_{2}$ genomes of $l_{s^{\prime}}(1,:)$.

\footnotetext{
${ }^{1}$ This genetic operator is heavily inspired by one of the very famous one roulette wheel selection.
}

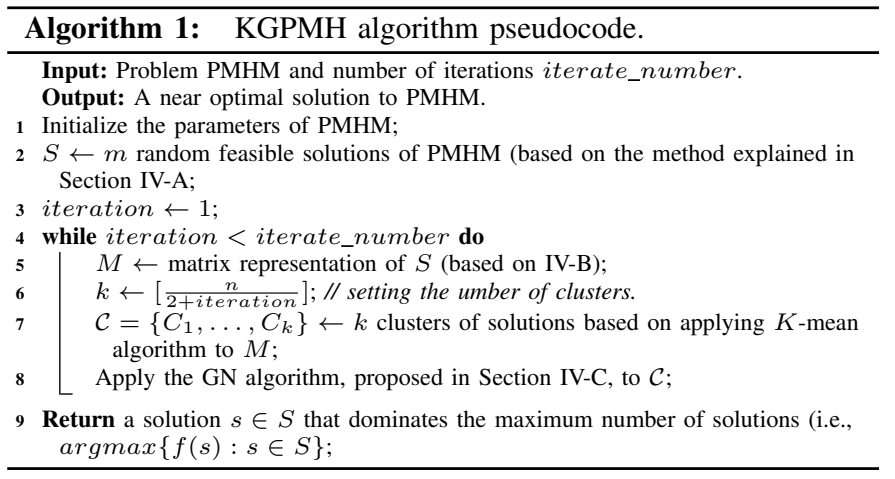

- Randomly choose $r_{1}$ locations of the $l_{s}(1,:)$ and $r_{2}$ locations of $l_{s^{\prime}}(1,:)$.

- Replace each of the selected location by a random integer numbers between 1 and $n$.

- Create two new solutions based on the new leader rows and the procedure introduced in Section IV-A.

- Add the obtained solutions to $S$.

\section{KGPMH algorithm}

Now by gathering all the components together, we are bale to depict our main algorithm in an integrated way. Our algorithm is named KGPMH, which stands for the KmeanGenetic-P-mobile-hub algorithm. Please note that the number of nodes $(n)$ and hubs $(p)$ must be fixed, known, and are considered as the inputs. In addition, point out that three periods are considered for the PMHM. Algorithm 1 elaborates on the description of KGPMH method.

\section{EXPERIMENTAL RESULTS}

In this section, we aim at evaluating our proposed technique by considering different ways. More specifically, in the sequel, we provide four analyses so as to examine the proposed method:

- We first investigate the role of the VRCE in improving the results.

- We compare our results with ones obtained by two other state-of-the-art clustering-based facility location algorithm.

- We compare our algorithm with two commonly used multi-objective meta-heuristic algorithms NSGA-II and MOPSO.

- We also compare our results with the (near) optimal and very accurate solutions obtained by the CPLEX 12.90 solver. We give comprehensive detail and comparison about the values of objective functions and CPU time (CT) spent, obtained by considering different numbers of hubs and initial solutions.

- We investigate the effect of considering the mobility of hubs by analyzing the sensitivity over the related parameters $C_{2}$ and $F_{2}$.

- We investigate the effect of considering service time on the total cost. 
Let us mention that the experimental dataset for this study is based on a well-known and commonly used Australia Post dataset (AP). This dataset consists of 200 data points and contains the coordinates of each data point. Furthermore, it also provides the demand between each pair of points (matrix $W)$.

One remaining argument before diving into our experiments is about setting the parameters' values. Let us note that the following parametrization is obtained by an experimental manner. For this characterization, we consider matrices $F 1, F 2, C 1$, $C 2$ in such a way that their entries are randomly selected with an uniform distribution from the intervals [200,600], $[10,15],[10,20]$ and $[20,40]$, respectively. In addition, we initialize $\Delta=[2 h, 2.5 h, 3 h], \sigma=[24 h, 24 h, 24 h], \alpha=0.2$, $\gamma=[0.7,0.85,1]$, and $\Gamma=[0.8 m, m, 1.2 m]$, where $m$ is average value of AP data. We also take three capacity and congestion levels into the account. In this fashion, $C=\{S, M, L\}$ symbolizes three levels of capacity, small, medium, and large, and besides, $G=\{1,2,3\}$ represents three congestion levels. We also let $\tau=0.3$ and $\tau_{g}=(1+\tau)^{g-1}$ such that $g \in G$. According to the availability of the coordinates, we are able to obtain the Euclidean distance between each pair of data points. We define the traveling time between each two data points by: $t_{i, j}=t . d_{i, j}$ where, $d_{i, j}$ is the Euclidean distance between $i$ and $j$ and $t$ is is a factor that depends on the numbers of data. It guarantees that the service time limit, which is considered around 24 hours for each period, is satisfied [14]. We respectively, refer to OFV1 and OFV2 as the first and second objective function's values.

We also point out that we have implemented our algorithm with $\mathrm{C}++$ programming language and used a computer system that contained the processor Intel(R) Core (TM) i5-7300U CPU @ 2.60GHz, $2712 \mathrm{Mhz}, 2$ Core(s), 4 Logical Processor(s) and $8 G B$ of $R A M$.

\section{A. Estimating the VRCE}

This part intends to show how well the proposed evaluation method, VRCE, can impact the results. To do so, we have solved the model with both VRCE and a simple evaluating manner. To be more precise, in the case of a non-clustering manner, we just used the domination, and so an elementary function $f$, which for any given solution $s, f(s)$ returns the number of solutions $s$ dominates, to evaluate solutions. Since, in this case, neither a vector representation was considered and nor a clustering algorithm was applied, the genetic algorithm would have only relied on choosing parent solutions based on their ranks, obtained by the naive function $f$. Fig. 3 (up) and Fig. 3 (down) respectively illustrate the first and the second objective function's values obtained when i) the proposed vector representation evaluation method is used (blue curve) and 2) when just the simple domination is applied (red curve). As can be seen, the results associated with the blue curves significantly outperform those presented by the red curve.
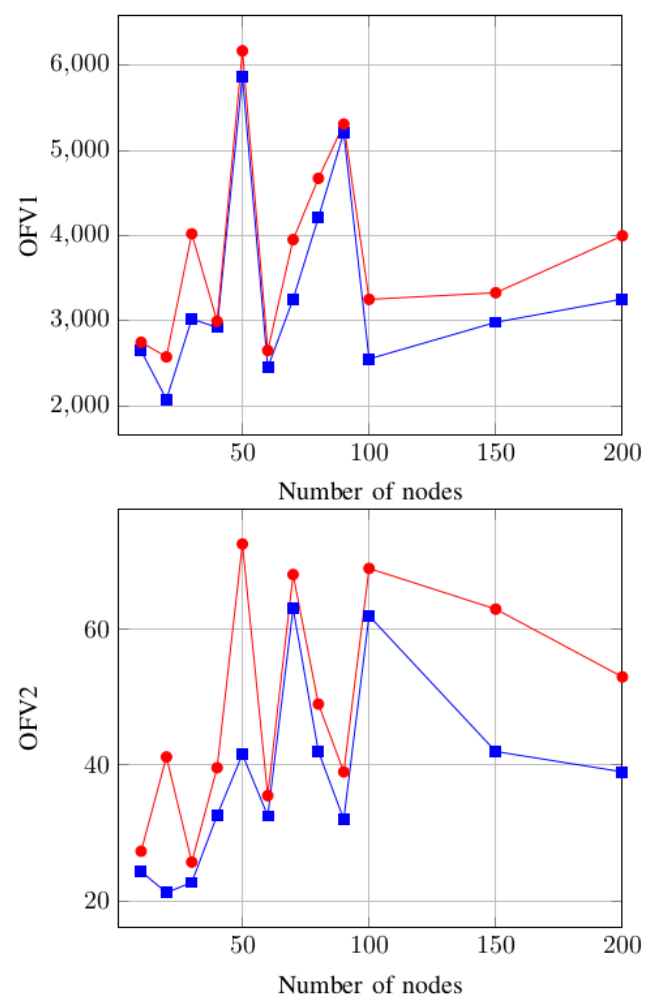

Fig. 3: (up) OFV1 and (down) OFV2 values obtained with and without considering the vector representation and clustering approach evaluation technique. The number of hubs is fixed and equal to 10. Furthermore, the number of initial solutions is equal to 15 , and the iteration number is 10 .

\section{B. Comparison with other clustering-based techniques}

We would have also liked to compare our results with those obtained by the two other clustering-based techniques: the CA-MOEA [45] and CRMO [44] ${ }^{2}$. CA-MOEA's idea is to replace the distance-based truncation style in NSGA-II with a hierarchical clustering technique (HCM) to cope with MOPs and MaOPs, respectively. This is while CRMO utilizes a series of predefined reference lines to bunch individuals into subregions so that each of the individuals nearest to the Pareto front will be chosen. Please note that these two algorithms can both perfectly be adapted to solve PMHP.

Therefore, same as what we did in the previous section, we again correspond two figures, which respectively present the first and the second objective functions' values obtained by KGPMH (blue curve) CA-MOEA (green curve) and CRMO (gray curve). Fig. 4 (up) and Fig. 4 (down) show the results. Observe that except in just one occasion regarding the second objective function, KGPMH results in obtaining less objective function values.

\footnotetext{
${ }^{2}$ In this paper, we coined the term $C R M O$ for the algorithm proposed in [44].
} 

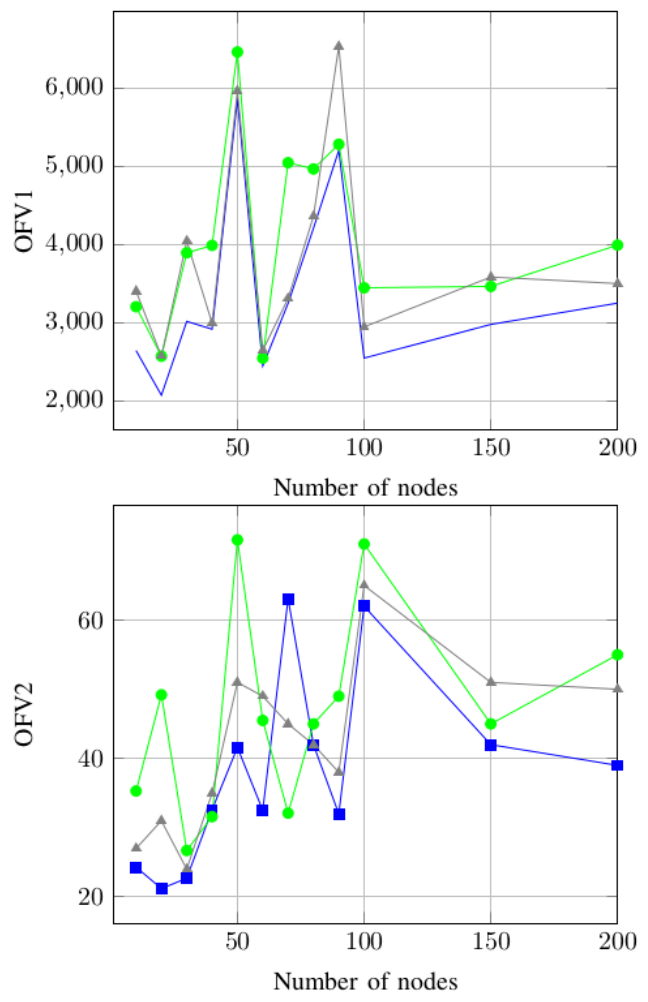

Fig. 4: (up) OFV1 and (down) OFV2 values obtained by KGPMH (blue curves), CA-MOEA (green curves), and CRMO (gray curve). The number of hubs is fixed and equal to 10. Furthermore, the number of initial solutions of KGPMH is equal to 15 , and the iteration number is 10 .

\section{Comparison with NSGA-II and MOPSO algorithms}

Let us start by briefly explaining the two well-used heuristic algorithms that can perfectly be applied to the context problem. We then assess their results against the ones obtained by our proposed technique.

NSGA-II is one of the most well-known algorithms for solving multi-objective problems proposed by [49] for the first time. By adding two operators: non-dominated sorting and crowding distance to the single-objective GA, the NSGAII is obtained. On the other hand, in the MOPSO, which is developed by [50], a swarm is made of particles in which each solution acts as a particle. This algorithm works based on the group's intelligence, which means every particle knows its prior best position and best position of the entire swarm. Each particle moves at a certain velocity in the feasible space of the problem to produce new solutions. Algorithm 2 and algorithm 3 respectively show the pseudocodes of NSGA-II and MOPSO algorithms.

To evaluate the efficiency and capability of our proposed algorithm, we compare the solutions obtained by KGPMH with these two algorithms. The results are presented in Table II. Please note that, Table I shows the parameters' values of KGPMH, NSGA-II, and MOPSO algorithms, which are set for the experiments.
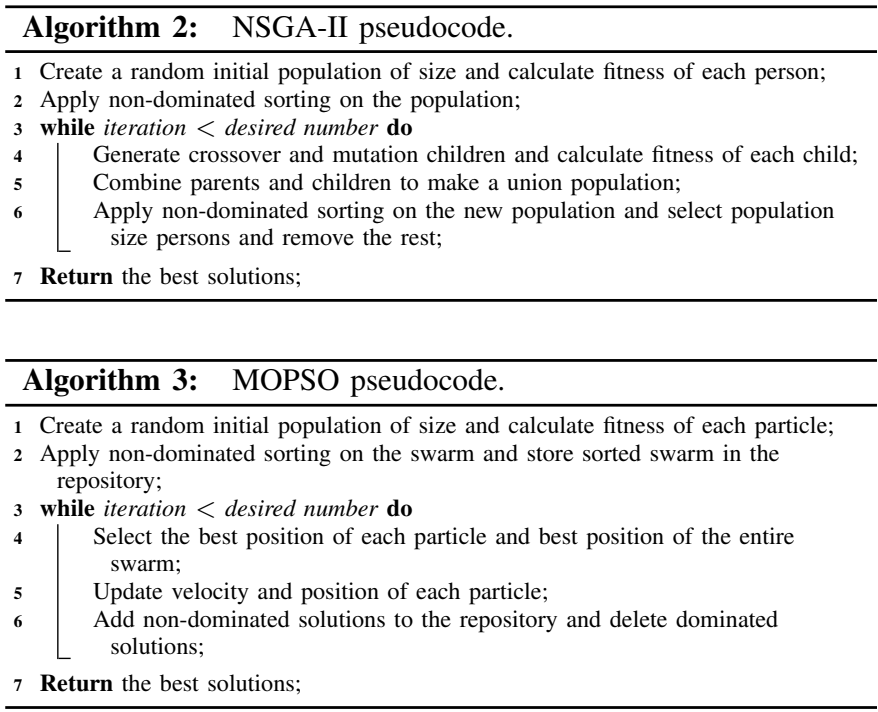

The results clearly illustrate that the KGPMH algorithm outperforms both NSGA-II and MOPSO algorithms in all cases in terms of detecting better solutions and spending less running time.

D. Comparison with (near) optimal Pareto solutions discovered by CPLEX solver

Although measuring the quality of our algorithm against two other triumphant and famous heuristic approaches raised promising results, it would considerably be satisfying to see the difference between the solutions obtained by the proposed algorithm and the optimal ones. Nevertheless, recall that a feasible solution that minimizes all objective functions simultaneously does not typically exist in our under-study bi-objective optimization problem. Therefore, by a solution optimal, one can refer to as Pareto optimal solutions: solutions that cannot be improved in any of the objectives without degrading at the other objectives. To obtain such solutions, we used the very robust solver CPLEX, which can perfectly solve PMHM and obtain the Pareto optimal solutions efficiently. Nevertheless, since the context problem is NP-hard, it is not very reassuring to expect achieving the Pareto optimal solutions by spending reasonable time. However, it is possible to determine these solutions by considering a smaller size (version) of the dataset.

In consequence, to compare our proposed algorithm's result with the Pareto optimal solutions, we have considered the AP dataset with 10 data points. Contemplating this number of nodes enables us to obtain the Pareto optimal solution using CPLEX solver, version 12.9.0, which perfectly offers new features to implement multi-objective optimization. This solver offers a mixture of blended and lexicographic (or hierarchical) optimization. A blended objective consists of simply the linear combination of several objectives with given weights. A lexicographic objective supposes that an order has been given among the various objective functions. This order enables us to define a lexicographic order among solutions: a 
TABLE I: MOPSO, NSGA-II and KGPMH parameters.

\begin{tabular}{lll} 
Algorithm & Parameter & Value \\
\hline MOPSO & Repository size & 75 \\
& Inertia weight & 0.6 \\
& Personal learning coefficient & 1.5 \\
& Global learning coefficient & 1 \\
& Population size & 90 \\
& Iteration & 70 \\
\hline NSGA-II & Crossover coefficient & 0.4 \\
& Mutation coefficient & 0.1 \\
& Population size & 90 \\
& Iteration & 70 \\
\hline KGPMH & Learning rate & 1 \\
& Time constant & 100 \\
& Population size & 90 \\
& Number of iteration & 70
\end{tabular}

solution is lexicographically smaller than another one if it is smaller in the first objective where they differ (following the order). An optimal solution is then one that is lexicographically minimal (or maximal depending on the optimization sense). Consequently, CPLEX can combine both blended and lexicographic objectives in the same optimization problem.

Table III presents the results obtained by applying KGPMH and also by using the CPLEX solver. By comparing the result, one can conspicuously observe that KGPMH's results exceed the ones obtained by CPLEX in almost every case, showing us the accuracy of the proposed method. Please note that the number of iteration in KGPMH in this experiment is equal to 15 .

\section{E. Effect of considering the mobility for hubs on total cost}

In this section, we are going to show how letting the hubs have mobility can decrease the total cost. For doing this, we have considered 20 data points of the AP dataset. We have also let $p=5$ and applied the KGPMH algorithm to the problem. Point out that the final obtained value of the first and second objective functions and the CPU time spent on this problem are respectively $3553,47.49$, and 16 seconds. Fig. 5 illustrates the network scheme that is derived based on the final solution. This is while when hubs' mobility is ignored, the total cost will be 3812 . Therefore, one might readily conclude that taking mobility into consideration can significantly help us decrease the total costs.

For a better understanding, recall from Section III-B that $C_{2}$ and $F_{2}$ are respectively the cost of constructing routes between hubs and the cost of transferring a mobile hub. We perform two separate sensitivity analyses over $C 2$ and $F 2$ to better analyze the effect of considering and not considering the mobility feature on total cost's value. More specifically, we show how much change will occur in total cost's value provided that we consider different multiples of $C_{2}$ and $F_{2}$ in the case that hubs have mobility. Table IV shows this issue. According to the results shown in Table IV and Fig. 6, it is apparent that whenever the cost of constructing routes between hubs $(C 2)$ and the cost of transferring a mobile hub $(F 2)$ are both lower than the cost of establishing a hub, the proposed model decides to move the hubs, while when the cost of establishing route and the cost of transferring a mobile hub are sufficiently high, the proposed model acts as a non-mobile model and establishes a hub instead of relocating hubs.

\section{F. The impact of considering service time}

Recall that service time is directly related to the variable ready time, which is the time spending at hubs for processing the flows. Based on our proposed model, one trivial consequence is that ready time also directly relates to the total cost.

Intuitively, it is natural to think that forcing hubs to process the flows and transportation faster requires spending more money. Table V, Fig. 7, and 8 visibly show this issue. As can be seen, the longer the service time is, the lower the total cost is.

Therefore, one can think of considering enough large value for service time can make us decrease the costs. Nevertheless, the critical argument, on the other hand, is that in real world, we have a time limit for sending each shipment on each transit network, and therefore, we must consider (obtain) a reasonable service time. As a result, considerably promising is to make a balance, or to be more precise, determine an appropriate value for the service time. In other words, we need to determine a proper service time that is feasible and also maintain the efficiency of a transit network.

We conclude this section by noting that our proposed method was able to surpass two of the state-of-the-art heuristic approaches and managed to achieve up-and-coming solutions near the optimal one. On the other hand, the novel consideration, mobility of hubs, allowed us to be capable of wisely decreasing the total cost. Furthermore, it turned out the decisive role of the service time in this problem.

\section{CONCLUSION}

This paper introduced a multi-period single-allocation $p$ mobile hub location problem with a service time limit. There are many real-world cases, such as chain stores in the supply chain, telecommunication systems, massage delivery systems, and post-delivery, which this problem can model. In our proposed model, each hub contained a specific capacity and congestion level. The aim was to design a transportation network for each period and select (i.e., locate) $p$ vertices to be hubs to satisfy all the demands with the minimum total cost, congestion, and ready time at hubs. In this fashion, the total cost was divided into transportation cost, construction of mobile infrastructure, establishing hubs, and transferring mobile hubs. The service time was the sum of time spent on gathering all the flows into the hub and the time spent at that hub for processing the flows. Also, ready time in each hub was the last arrival time of all flows from nonhub nodes to that hub vertex. Besides modeling the problem, this paper proposed a novel hybrid meta-heuristic method for solving the problem. We devised a novel function for evaluating solutions, and based on that, we provided a vector representation for each solution. We then employed the Kmeans clustering algorithm and a genetic approach to evaluate and generate solutions. We finally measured the quality of our algorithm against several other state-of-the-art facility location algorithms. The results proved this claim that our proposed method is a promising alternative for all the comparable algorithms. We also discussed the pivotal roles that our two 
TABLE II: Computational results of the comparison between KGPMH, NSGA-II, and MOPSO algorithms.

\begin{tabular}{|c|c|c|c|c|c|c|c|c|c|c|c|c|c|}
\hline \multirow{3}{*}{ \# of nodes } & \multicolumn{3}{|c|}{ KGPMH } & \multicolumn{3}{|c|}{ NSGA-II } & \multicolumn{2}{|c|}{ Gap (\%) } & \multicolumn{3}{|c|}{ MOPSO } & \multicolumn{2}{|c|}{ Gap (\%) } \\
\hline & OVF1 & OVF2 & $\mathrm{CT}(\mathrm{s})$ & OVF1 & OVF2 & $\mathrm{CT}(\mathrm{s})$ & OVF1 & OVF2 & OVF1 & OVF2 & $\mathrm{CT}(\mathrm{s})$ & OVF1 & OVF2 \\
\hline & 3693 & 39.4 & 5.3 & 3906 & 42.9 & 9.5 & 3.8 & 3.6 & 3958 & 42.6 & 8.7 & 5.1 & 2.8 \\
\hline 15 & 4581 & 39.99 & 7.9 & 5183 & 44.85 & 16.8 & 5.5 & 2.8 & 5091 & 4512 & 15.47 & 3.6 & 3.4 \\
\hline 20 & 59072 & 43.17 & 16 & 6557 & 49.03 & 34 & 7.8 & 3.2 & 6636 & 49.53 & 29 & 9.1 & 4.2 \\
\hline 25 & 8119 & 57.98 & 27 & 8754 & 66.1 & 56.2 & 6.6 & 1.8 & 8627 & 67.9 & 65.8 & 5.1 & 1.5 \\
\hline 30 & 17171 & 94.83 & 1024.38 & 18305 & 109.08 & 1907.41 & 4.4 & 2.6 & 18678 & 110.13 & 1843.41 & 6.6 & 3.6 \\
\hline 35 & 19124 & 103.5 & 1091.3 & 21007 & 151.17 & 2001.34 & 6.7 & 4.8 & 21325 & 152.65 & 1900.43 & 8.3 & 5.9 \\
\hline 40 & 19512 & 168.4 & 1555.03 & 23741 & 169.83 & 2982.4 & 6.9 & 5.1 & 22435 & 171.56 & 2876.9 & 5.6 & 6.2 \\
\hline 80 & 66781 & 403.73 & 150201.45 & 84512 & 691.25 & 312078.9 & 5.8 & 3.4 & 81413 & 681.13 & 301121.4 & 6.2 & 5.9 \\
\hline 110 & 108439 & 925.67 & 84512.14 & 145970 & 1258.39 & 897514.1 & 4.1 & 2.2 & 142432 & 1021.83 & 890181.7 & 5.9 & 4.8 \\
\hline
\end{tabular}

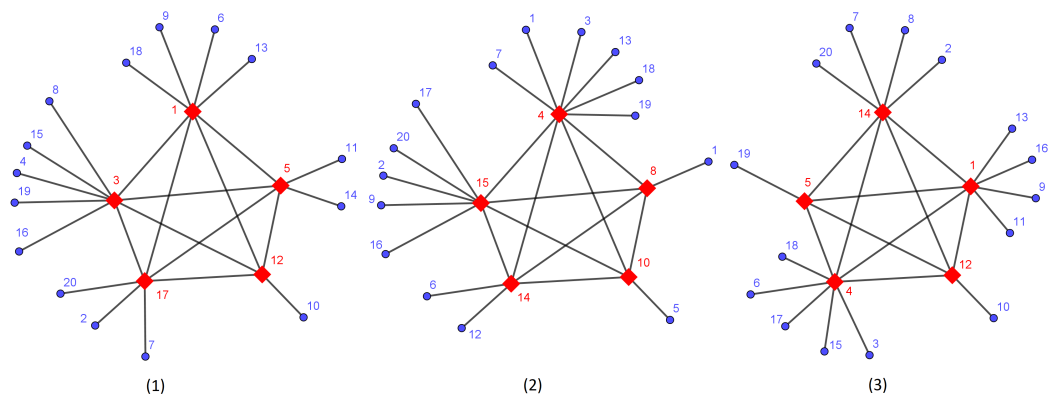

Fig. 5: (1), (2) and (3) show the network scheme of the AP dataset with considering 20 data points, in period 1, 2 and 3, respectively. Red nodes are hubs.

TABLE III: Objective functions' values and CT spent by applying KGPMH algorithm to the AP dataset with 10 data points and also the Pareto optimal solutions' values obtained by Cplex solver.

\begin{tabular}{|c|c|c|c|c|c|c|}
\hline \# hubs & $\begin{array}{l}\text { \# initial random } \\
\text { solutions }\end{array}$ & $\begin{array}{c}\text { OFV1 } \\
\text { (KGPMH) }\end{array}$ & $\begin{array}{c}\text { OVF2 } \\
\text { (KGPMH) }\end{array}$ & $\mathrm{CT}(\mathrm{s})$ & $\begin{array}{l}\text { OVF1 } \\
\text { (Cplex) }\end{array}$ & $\begin{array}{l}\text { OVF2 } \\
\text { (Cplex) }\end{array}$ \\
\hline 2 & 4 & 2659.12 & 24.4 & 2.9 & 2661.55 & \\
\hline 2 & 8 & 2247.91 & 21.2 & 4.5 & 2279.53 & 23.08 \\
\hline 2 & 15 & 2085.124 & 21.14 & 7.6 & 2096.72 & 20.06 \\
\hline 3 & 4 & 3152.12 & 29.3 & 2.9 & 3123.73 & 31.6 \\
\hline 3 & 8 & 3054.45 & 28.6 & 5.5 & 2883.3 & 26.9 \\
\hline 3 & 15 & 3001.674 & 26.2 & 12.3 & 3254.75 & 29.01 \\
\hline 4 & 4 & 3763.175 & 41.4 & 5.3 & 3812.321 & 45.3 \\
\hline 4 & 8 & 3690.53 & 20.1 & 8.6 & 3341 & 19.81 \\
\hline 4 & 25 & 2706.53 & 22.8 & 15.4 & 3214.63 & 27 \\
\hline
\end{tabular}

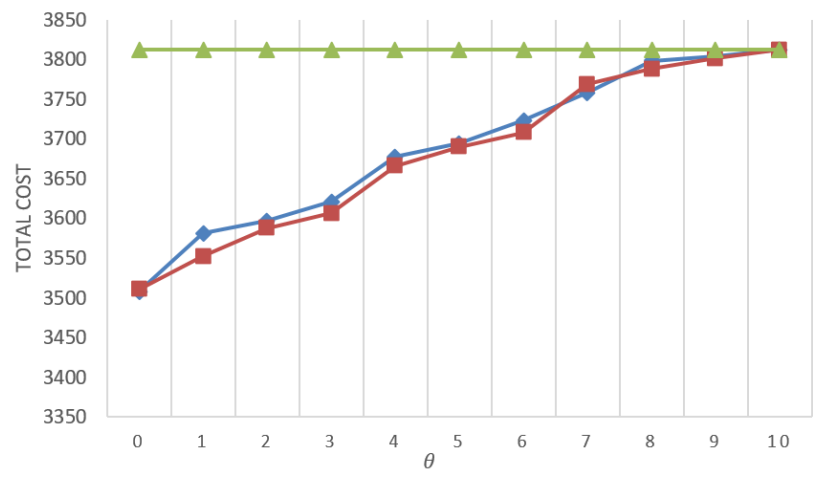

Fig. 6: Comparison of the network total cost with and without considering hubs mobility. Blue and red diagrams respectively show the impress of different multiples of $F 2$ and $C 2$ in the case that hubs have mobility. Green diagram shows the nonmobile condition.

simultaneous considerable factors, service time and mobility of hubs, play.

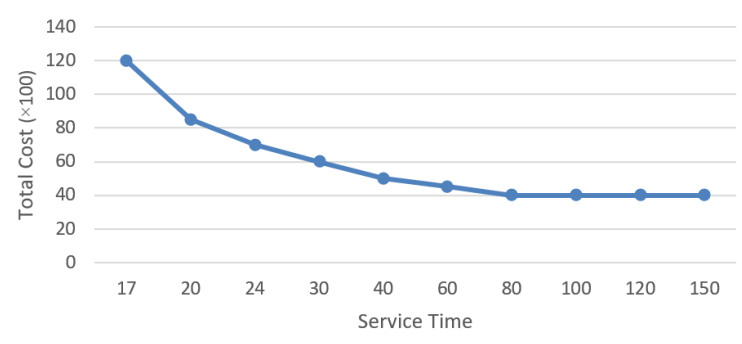

Fig. 7: Effects of service time on total cost.

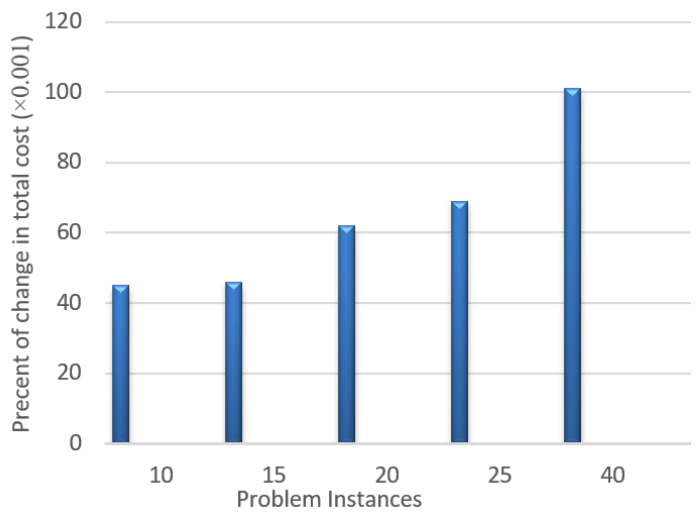

Fig. 8: Comparison of costs with and without service time.

\section{CONFLICT OF INTEREST}

The authors declare that there is no potential conflict of interest regarding the publication of this paper.

\section{ETHICAL APPROVAL}

The authors certify that they have no affiliation with or involvement with human participants or animals performed 
TABLE IV: Sensitivity analysis over $C 2$ and $F 2$. Different value of cost values provided that different multiple of $C_{2}$ and $F_{2}$ are considered. $\theta$ represents the multiple of the parameters $C 2$ and $F 2$

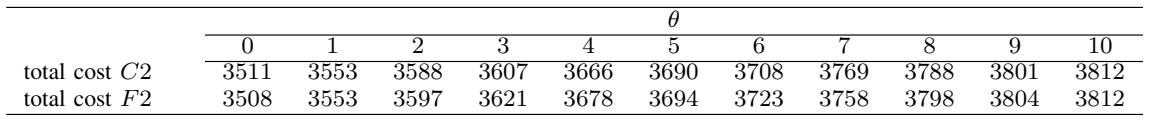

TABLE V: Comparison of costs with and without the service time.

\begin{tabular}{|c|c|c|c|c|c|c|}
\hline & \multicolumn{3}{|c|}{$\begin{array}{l}\text { PMHM with } \\
\text { service time }\end{array}$} & \multicolumn{3}{|c|}{$\begin{array}{c}\text { PMHM without } \\
\text { service time }\end{array}$} \\
\hline \# nodes & OFV1 & CT (s) & \# hubs & OVF1 & CT (s) & \# hubs \\
\hline 10 & 3763 & 5.3 & 4 & 3593 & 4.8 & 3 \\
\hline 15 & 4912 & 7.9 & 4 & 4687 & 7.6 & 4 \\
\hline 20 & 6082 & 16 & 5 & 5701 & 14.7 & 5 \\
\hline 25 & 8212 & 27 & 6 & 7641 & 24.5 & 7 \\
\hline 40 & 18388 & 155.03 & 14 & 16523 & 141.31 & 12 \\
\hline
\end{tabular}

by any of the authors in any organization or entity with any financial or non-financial interest in the subject matter or materials discussed in this paper.

\section{REFERENCES}

[1] A. Bruns, A. Klose, and P. Stähly, "Restructuring of swiss parcel delivery services," OR-Spektrum, vol. 22, no. 2, pp. 285-302, 2000.

[2] H. Tikani, M. Honarvar, and Y. Z. Mehrjerdi, "Developing an integrated hub location and revenue management model considering multi-classes of customers in the airline industry," Computational and Applied Mathematics, vol. 37, no. 3, pp. 3334-3364, 2018.

[3] P. Memari, R. Tavakkoli-Moghaddam, F. Navazi, and F. Jolai, "Air and ground ambulance location-allocation-routing problem for designing a temporary emergency management system after a disaster," Proceedings of the Institution of Mechanical Engineers, Part H: Journal of engineering in medicine, p. 0954411920925207, 2020.

[4] R. Kamoshida, "Concurrent optimization of job shop scheduling and dynamic and flexible facility layout planning," in 2018 5th International Conference on Industrial Engineering and Applications (ICIEA). IEEE, 2018, pp. 289-293.

[5] P. Benalcazar, J. Kamiński, and P. W. Saługa, "The storage location problem in a coal supply chain: background and methodological approach," Gospodarka Surowcami Mineralnymi, vol. 33, 2017.

[6] D. Krivitski, A. Schuster, and R. Wolff, "A local facility location algorithm for sensor networks," in International Conference on Distributed Computing in Sensor Systems. Springer, 2005, pp. 368-375.

[7] A. Ferdowsi, A. Khanteymoori, and M. D. Chenary, "Tscda: A novel greedy approach for community discovery in networks," bioRxiv, 2021

[8] R. Mahapatra, S. Samanta, T. Allahviranloo, and M. Pal, "Radio fuzzy graphs and assignment of frequency in radio stations," Computational and Applied Mathematics, vol. 38, no. 3, p. 117, 2019.

[9] R. Rahmaniani and A. Ghaderi, "A combined facility location and network design problem with multi-type of capacitated links," Applied Mathematical Modelling, vol. 37, no. 9, pp. 6400-6414, 2013.

[10] H. Karimi and M. Setak, "Flow shipment scheduling in an incomplete hub location-routing network design problem," Computational and Applied Mathematics, vol. 37, no. 2, pp. 819-851, 2018.

[11] D. D. Čvokić and Z. Stanimirović, "A single allocation hub location and pricing problem," Computational and Applied Mathematics, vol. 39, no. 1, pp. 1-24, 2020.

[12] A. B. Arabani and R. Z. Farahani, "Facility location dynamics: An overview of classifications and applications," Computers \& Industrial Engineering, vol. 62, no. 1, pp. 408-420, 2012.

[13] G. Ahmadi, R. Tavakkoli-Moghaddam, A. Baboli, and M. Najafi, "A decision support model for robust allocation and routing of search and rescue resources after earthquake: a case study," Operational Research, pp. 1-43, 2020.

[14] S. A. Alumur, S. Nickel, B. Rohrbeck, and F. Saldanha-da Gama, "Modeling congestion and service time in hub location problems," Applied Mathematical Modelling, vol. 55, pp. 13-32, 2018.
[15] M. E. O'kelly, "A quadratic integer program for the location of interacting hub facilities," European journal of operational research, vol. 32, no. 3, pp. 393-404, 1987.

[16] J. F. Campbell, "Integer programming formulations of discrete hub location problems," European Journal of Operational Research, vol. 72 , no. 2, pp. 387-405, 1994.

[17] I. Correia, S. Nickel, and F. Saldanha-da Gama, "Single-assignment hub location problems with multiple capacity levels," Transportation Research Part B: Methodological, vol. 44, no. 8-9, pp. 1047-1066, 2010.

[18] P. G. Grove and M. E. O'Kelly, "Hub networks and simulated schedule delay," Papers in Regional Science, vol. 59, no. 1, pp. 103-119, 1986.

[19] M. Karimi-Mamaghan, M. Mohammadi, A. Pirayesh, A. M. KarimiMamaghan, and H. Irani, "Hub-and-spoke network design under congestion: A learning based metaheuristic," Transportation Research Part E: Logistics and Transportation Review, vol. 142, p. 102069, 2020.

[20] A. Ghodratnama, H. Arbabi, and A. Azaron, "A bi-objective hub location-allocation model considering congestion," Operational Research, vol. 20, no. 4, pp. 2427-2466, 2020.

[21] V. Rodriguez, M. Alvarez, and L. Barcos, "Hub location under capacity constraints," Transportation Research Part E: Logistics and Transportation Review, vol. 43, no. 5, pp. 495-505, 2007.

[22] M. T. Melo, S. Nickel, and F. S. Da Gama, "Dynamic multi-commodity capacitated facility location: a mathematical modeling framework for strategic supply chain planning," Computers \& Operations Research, vol. 33, no. 1, pp. 181-208, 2006.

[23] S. A. Alumur, S. Nickel, F. Saldanha-da Gama, and Y. Seçerdin, "Multiperiod hub network design problems with modular capacities," Annals of Operations Research, vol. 246, no. 1-2, pp. 289-312, 2016.

[24] I. Correia, S. Nickel, and F. Saldanha-da Gama, "A stochastic multiperiod capacitated multiple allocation hub location problem: Formulation and inequalities," Omega, vol. 74, pp. 122-134, 2018.

[25] R. Halper and S. Raghavan, "The mobile facility routing problem," Transportation Science, vol. 45, no. 3, pp. 413-434, 2011.

[26] C. Lei, W.-H. Lin, and L. Miao, "A multicut 1-shaped based algorithm to solve a stochastic programming model for the mobile facility routing and scheduling problem," European Journal of operational research, vol. 238, no. 3, pp. 699-710, 2014.

[27] H. Güden and H. Süral, "Locating mobile facilities in railway construction management," Omega, vol. 45, pp. 71-79, 2014.

[28] M. Bashiri, M. Rezanezhad, R. Tavakkoli-Moghaddam, and H. Hasanzadeh, "Mathematical modeling for a p-mobile hub location problem in a dynamic environment by a genetic algorithm," Applied Mathematical Modelling, vol. 54, pp. 151-169, 2018.

[29] M. Qi, C. Cheng, X. Wang, and W. Rao, "Mobile facility routing problem with service-time-related demand," in 2017 International Conference on Service Systems and Service Management. IEEE, 2017, pp. $1-6$.

[30] M. Mokhtarzadeh, R. Tavakkoli-Moghaddam, C. Triki, and Y. Rahimi, "A hybrid of clustering and meta-heuristic algorithms to solve a p-mobile hub location-allocation problem with the depreciation cost of hub facilities," Engineering Applications of Artificial Intelligence, vol. 98, p. 104121, 2021.

[31] C. S. ReVelle and H. A. Eiselt, "Location analysis: A synthesis and survey," European journal of operational research, vol. 165, no. 1, pp. $1-19,2005$.

[32] R. Z. Farahani, M. SteadieSeifi, and N. Asgari, "Multiple criteria facility location problems: A survey," Applied mathematical modelling, vol. 34, no. 7, pp. 1689-1709, 2010.

[33] A. Ahmadi-Javid, P. Seyedi, and S. S. Syam, "A survey of healthcare facility location," Computers \& Operations Research, vol. 79, pp. 223 263, 2017.

[34] P. Cappanera, "A survey on obnoxious facility location problems," 1999.

[35] D.-w. Tcha and B.-i. Lee, "A branch-and-bound algorithm for the multilevel uncapacitated facility location problem," European Journal of Operational Research, vol. 18, no. 1, pp. 35-43, 1984. 
[36] H. Mostafaei, Y. Alipouri, and J. Shokri, "A mixed-integer linear programming for scheduling a multi-product pipeline with dual-purpose terminals," Computational and Applied Mathematics, vol. 34, no. 3, pp. 979-1007, 2015.

[37] C. Ortiz-Astorquiza, I. Contreras, and G. Laporte, "An exact algorithm for multilevel uncapacitated facility location," Transportation Science, vol. 53, no. 4, pp. 1085-1106, 2019.

[38] E. Gimadi, A. Shtepa, and O. Tsidulko, "Improved exact algorithm for the capacitated facility location problem on a line graph," in 2019 15th International Asian School-Seminar Optimization Problems of Complex Systems (OPCS). IEEE, 2019, pp. 53-57.

[39] D. C. Turkoglu and M. E. Genevois, "A comparative survey of service facility location problems," Annals of Operations Research, pp. 1-70, 2019.

[40] S.-Y. Hsieh and S.-S. Kao, "A survey of hub location problems," Journal of Interconnection Networks, vol. 19, no. 01, p. 1940005, 2019.

[41] A. S. Barjouei, A. Barabadi, and R. Tavakkoli-Moghaddam, "A goal programming approach for a fuzzy single-source capacitated facility location problem," in 2019 IEEE International Conference on Industrial Engineering and Engineering Management (IEEM). IEEE, 2019, pp. $1388-1392$.

[42] T. Hiroyasu, M. Miki, and S. Watanabe, "The new model of parallel genetic algorithm in multi-objective optimization problems-divided range multi-objective genetic algorithm," in Proceedings of the 2000 Congress on Evolutionary Computation. CECOO (Cat. No. 00TH8512), vol. 1. IEEE, 2000, pp. 333-340.

[43] Y. Jin, M. Olhofer, and B. Sendhoff, "Dynamic weighted aggregation for evolutionary multi-objective optimization: Why does it work and how?" in Proceedings of the Genetic and Evolutionary Computation Conference (GECCO2001), 2001, pp. 1042-1049.

[44] L. Cai, S. Qu, Y. Yuan, and X. Yao, "A clustering-ranking method for many-objective optimization," Applied Soft Computing, vol. 35, pp. 681694, 2015.

[45] Y. Hua, Y. Jin, and K. Hao, "A clustering-based adaptive evolutionary algorithm for multiobjective optimization with irregular pareto fronts," IEEE Transactions on Cybernetics, vol. 49, no. 7, pp. 2758-2770, 2018

[46] D. Celik Turkoglu and M. Erol Genevois, "A comparative survey of service facility location problems," Annals of Operations Research, vol. 292, no. 1, pp. 399-468, 2020.

[47] K. Deb, "Multi-objective optimisation using evolutionary algorithms: an introduction," in Multi-objective evolutionary optimisation for product design and manufacturing. Springer, 2011, pp. 3-34.

[48] H. O. Lancaster and E. Seneta, "Chi-square distribution," Encyclopedia of biostatistics, vol. 2, 2005.

[49] N. Srinivas and K. Deb, "Muiltiobjective optimization using nondominated sorting in genetic algorithms," Evolutionary computation, vol. 2, no. 3, pp. 221-248, 1994.

[50] C. A. C. Coello, G. T. Pulido, and M. S. Lechuga, "Handling multiple objectives with particle swarm optimization," IEEE Transactions on evolutionary computation, vol. 8, no. 3, pp. 256-279, 2004. 